\title{
NUMERICAL ANALYSIS OF WAVE PHENOMENA IN HYPERELASTIC MOONEY-RIVLIN AND ZAHORSKI MATERIALS
}

\author{
Izabela MAJOR ${ }^{1}$ \\ ${ }^{1}$ Department of Applied Mechanics, Faculty of Civil Engineering, Czestochowa University of \\ Technology, Czestochowa, Poland. \\ "corresponding author: admin@major.strefa.pl, +48 343250965.
}

\section{Abstract}

This paper focuses on presentation of waves phenomena that occur during propagation of disturbance in continuous incompressible hyperelastic structures, described with elastic potential. Numerical analysis demonstrated differences during the propagation of disturbances in the commonly used model of Mooney-Rivlin material compared to less popular Zahorski material. The obtained result is also likely to contribute to development of new forms of practical application of nonlinear rubber and rubber-like materials for technological solutions, including those used in the broadly understood construction sector.
\end{abstract}

\author{
Keywords: \\ FEM models; \\ Hyperelastic materials; \\ Mooney-rivlin material; \\ Numerical analysis: \\ Zahorski material; \\ Wave propagation.
}

\section{Introduction}

The wave phenomena that occur in the materials with particular elastic potential, i.e. elastic in Green's terms, should be regarded as propagation of waves of discontinuities and travelling waves in the material continuum modelled as nonlinear isotropic elastic medium, which is both compressible and incompressible. Process of propagation of a disturbance in a material continuum modelled as a moving surface of discontinuities is one of the most difficult problems of contemporary continuum mechanics. With this approach, the propagating wave represents a disturbance which is strictly reduced to the surface. The advantage of this concept lies in the opportunities for considering wave phenomena from the standpoint of a relatively uniform theory that does not require any simplification assumptions. The constantly improving methods of determination of elastic constants for compressible and incompressible materials offer increasingly better opportunities for a more comprehensive experimental analysis. The data obtained with these analyses are necessary for development of modern measurement techniques and modelling the processes that occur due to propagation of disturbance in a continuum. Furthermore, they are extremely useful for formulation and adopting physical assumptions for determination of a mathematical model of a body and are used for verification of the correctness of the calculations. Knowledge about wave phenomena that concern the problems of propagation of the surfaces of discontinuities in the material continuum has many practical implications. Demonstration of the differences that occur during propagation of disturbance in the commonly used model of Mooney-Rivlin material compared to less popular Zahorski material is also likely to contribute to development of new forms of practical application of nonlinear rubber and rubber-like materials for technological solutions, including those used in the broadly understood construction sector.

\section{Constitutive equations of hyperelastic materials - general information}

Behaviour of a particular material caused by various external factors is described by means of the constitutive equations which are also termed physical relationships for a particular material medium. Physical relationships that describe behaviour of materials subjected to deformations have been investigated since the beginning of the $20^{\text {th }}$ century. Further, in the forties and fifties, the first attempts were also made to determine the constitutive relationships that describe behaviour of rubber and rubber-like materials [1], [2]. A general form of the function of elastic energy was obtained in 1951 
by Rivlin and Saunders [3]. Based on the studies above, rubber-like materials are considered as incompressible. The function of deformation energy for these materials depends on two invariants of the deformation tensor and on two constants. Describing the elastic behaviour of rubber-like materials with substantial deformations became possible after introduction of the function of deformation energy by Zahorski in 1959 [4], with incompressible material described by nonlinear dependency on the invariants of deformation tensor.

Constitutive equations that describe the relationships between deformations and stresses or between deformations and energy for hyperelastic materials are obtained based on the equations of mechanical energy balance. These relationships have the nature of phenomenological relations determined usually by means of experimental studies and represent a specific link that connects deformation of the material caused by the stress. The model of material is adopted depending on the factors that are of essential importance to behaviour of the specific medium. Therefore, constitutive equations define an arbitrary selected model of material which describes its actual behaviour (better or worse) in a particular are of changes in these factors. In terms of the theory of elasticity and in broadly understood mechanical problems, including continuum mechanics, elastic bodies are considered as material continuum with internal bonds and without them. For the elastic bodies without bonds, the properties of such a medium are given if the function $W$ can be defined, which, for any deformation $d$ of this medium, determines the corresponding elastic energy $W=W(d)$ accumulated in the unit of volume with respect to the reference configuration $\mathscr{B}_{\mathscr{R}}$. Function $W$ is typically defined as a function of deformation energy.

For uniform isotropic elastic bodies, the constitutive equations can be written as

$W=W\left(I_{1}, I_{2}, I_{3}\right)$,

where $I_{1}, I_{2}, I_{3}$ are invariants of the deformation tensor.

An elastic body with imposed internal bonds (with its most significant form being incompressibility) cannot be subjected to any deformations. The only acceptable deformations with regard to incompressible bodies are deformations which do not change its volume (isochoric), which have to meet the following condition

$$
I_{3}=1
$$

thus, deformation energy for an incompressible body represents a function of only two other invariants. This can be re-written in a form which is analogous to the condition (1)

$W=W\left(I_{1}, I_{2}\right)$.

The equations (2) and (3) define the constitutive relations for incompressible material.

With large deformations, each of the rubber-like materials (natural or synthetic) behaves in a specific manner. Therefore, it is necessary to determine, for each individual case of experimental procedure, a model of constitutive equation, because one cannot adopt one generalized relationship which would suit different problems.

\section{Model of Zahorski material}

Model of Zahorski material is described by the equation with nonlinear dependency on the invariants of the deformation tensor. This description determines behaviour of rubber at significantly greater deformations compared to neo-Hookean or Mooney-Rivlin material, whereas constitutive relationship for this material adopts the form

$$
\begin{aligned}
& W=W\left(I_{1}, I_{2}\right)=\Sigma \rho_{R}= \\
& =C_{1}\left(I_{1}-3\right)+C_{2}\left(I_{2}-3\right)+C_{3}\left(I_{1}^{2}-9\right),
\end{aligned}
$$


where

$W \quad$ - specific elastic energy in the configuration $\mathscr{B}_{\mathscr{R}}$,

$\Sigma \quad$ - elastic energy accumulated per mass unit,

$\rho_{R} \quad$ - mass density in the reference configuration $\mathscr{B}_{\mathscr{R}}$,

$I_{1}, I_{2} \quad$ - invariants of the deformation tensor,

$C_{1}, C_{2}, C_{3}$ - material constants.

The first and the second derivative of the function $\Sigma$ with respect to the invariants of the deformation tensor

$\Sigma_{1}=\frac{1}{\rho_{R}}\left(C_{1}+2 C_{3} I_{1}\right), \quad \Sigma_{2}=\frac{C_{2}}{\rho_{R}}, \quad \Sigma_{11}=\frac{2 C_{3}}{\rho_{R}}$.

Other derivatives are

$\Sigma_{22}=\Sigma_{12}=\Sigma_{21}=0$.

If the constant $\mathrm{C}_{3}=0$, the Mooney-Rivlin material is obtained

$W\left(I_{1}, I_{2}\right)=\Sigma \rho_{R}=C_{1}\left(I_{1}-3\right)+C_{2}\left(I_{2}-3\right)$.

The Table 1 below presents elastic constants for three grades of rubber. The values presented in the Table 1 are based on the study [5]. The values of the constants were obtained by computing per SI system units.

Table 1: Material constants for three kinds of rubber.

\begin{tabular}{|c|c|c|c|}
\hline Constants & $\begin{array}{c}\text { Rubber "A" } \\
{[\mathrm{Pa}]}\end{array}$ & $\begin{array}{c}\text { Rubber "B" } \\
{[\mathrm{Pa}]}\end{array}$ & $\begin{array}{c}\text { Rubber "C" } \\
{[\mathrm{Pa}]}\end{array}$ \\
\hline$C_{1}$ & $6.278 \cdot 10^{4}$ & $2.099 \cdot 10^{5}$ & $3.453 \cdot 10^{5}$ \\
\hline$C_{2}$ & $8.829 \cdot 10^{3}$ & $1.275 \cdot 10^{4}$ & 0 \\
\hline$C_{3}$ & $6.867 \cdot 10^{3}$ & $3.924 \cdot 10^{3}$ & $2.256 \cdot 10^{4}$ \\
\hline
\end{tabular}

For the rubber analysed in this study: "A" (hardness $50-51^{\circ} \mathrm{Sh}$ ), "B" (hardness $55-56^{\circ} \mathrm{Sh}$ ) and "C" (hardness $73-74^{\circ} \mathrm{Sh}$ ), the following content of the mixture (see [5]).

The constitutive equation (4) allows for a more comprehensive analysis of the wave phenomena that propagate in the elastic incompressible materials with respect to Mooney-Rivlin material. A description that describes the behaviour of rubber with greater deformations was obtained. For the main elongation, this is even true for $\lambda=3$, whereas in neo-Hookean and Mooney-Rivlin materials, the acceptable results are obtained for $\lambda=1.4$ [6].

The nonlinear term $C_{3}\left(I_{1}^{2}-9\right)$ present in the equation (4) allows for an in-depth analysis and introduction of other qualitative elements for the description of wave processes. The relationship (4) models the effects of dynamic behaviour of materials and is used for the analysis of wave phenomena concerning propagation of the disturbance in the form of shock waves, travelling waves and solitary waves ([7], [8], [9], et al.)

\section{Model description and aim of the study}

For the numerical modelling of the distribution of velocities and stresses generated with the waves of discontinuities adopted by the $2 \mathrm{D}$, being the longitudinal cross-section 
with the length of $1 \mathrm{~m}$ (Fig. 1). Initial and final height of the longitudinal section of a round rod are $10 \mathrm{~cm}$. The load of the rod analysed was forced linear dislocation of the edges L3 towards the $y$ axis. The value of the forced displacement amounted to $10 \mathrm{~cm}$ for the time $t=0.1045 \mathrm{~s}$, which is consistent with the literature (see [10]). On the opposite (with respect to the load) L1 edge, the bonds were imposed to prevent from displacement towards $y$ and $z$ axes. These are bonds $\{B\}$, see Fig. 1. Discretization of the object discussed was carried out by means of 4 nodes. In general, 1000 flat elements (2D) we obtained for the longitudinal cross-section of the rod analysed.

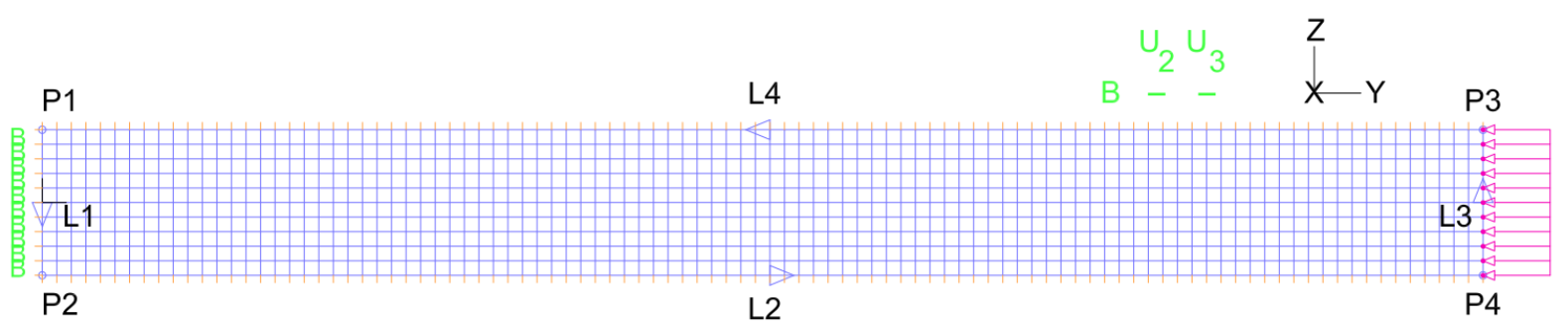

Fig. Chyba! Dokument neobsahuje žiadny text so zadaným štýlom.1: Model of incompressible rod in 2D: grid, load, boundary conditions and system of coordinates.

The aim of the numerical calculations was to compare the distribution of velocities and states of stress in Mooney-Rivlin material and Zahorski material for the above boundary conditions and assumed deformations of the object that simulate uniaxial compression. Analysis of the state of stresses in the nonlinear elastic mass made of two different materials seems to be the most convenient form of presentation of differences in the distribution of stresses and velocities that result from the differences adopted for the numerical analysis of the models of materials. In this range, we focus on the study [11], where, also in the form of distribution of stress, the results of the numerical studies were presented. The declared load in the object occurred stepwise at the instant of $t=0.1045 \mathrm{~s}$. The process of propagation of the disturbance in the model could be observed in 100 other steps. Therefore, we obtained information about generation of the velocity of disturbance propagation in homogeneous incompressible rods made of two different hyperelastic materials.

\section{Comparative analysis of the results obtained for Mooney-rivlin and Zahorski materials}

For the boundary conditions studied and external effects (deformations), Fig. 2 and Fig. 3 present the course of disturbance propagation in the rod for selected time steps, and illustrate the distributions of the velocities and states of stresses for the rod made of two different hyperelastic materials.

\section{Conclusions}

The comparative analysis of distribution of the velocities and states of effective stress for the adopted model revealed insignificant differences in the distribution of both velocity and the effective stress obtained for Zahorski and Mooney-Rivlin material. These differences result from the elastic potential adopted for the materials analysed.

Fig. 2. illustrates distributions of velocities in the rubber "A" for vertical cross-section of the rod discussed. Insignificant differences in the distribution of velocity can be observed at the surface of the object studied. Mooney-Rivlin material has areas with the velocities higher than in the Zahorski material, Fig. 4.

Fig. 3 illustrates distributions of stresses in the rubber "A" for vertical cross-section of the rod discussed. Insignificant difference in the distribution of effective stresses can also be observed at the surface of the object studied. Mooney-Rivlin material has areas with the stresses higher than in the Zahorski material, Fig. 5. 
Step 90 - the top figure is Mooney - Rivlin material, the second is Zahorski material.
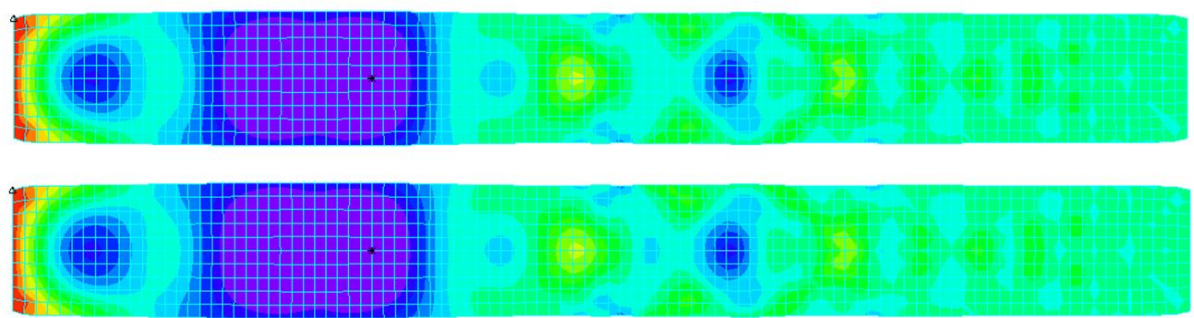

Step 100 - the top figure is Mooney - Rivlin material, the second is Zahorski material.
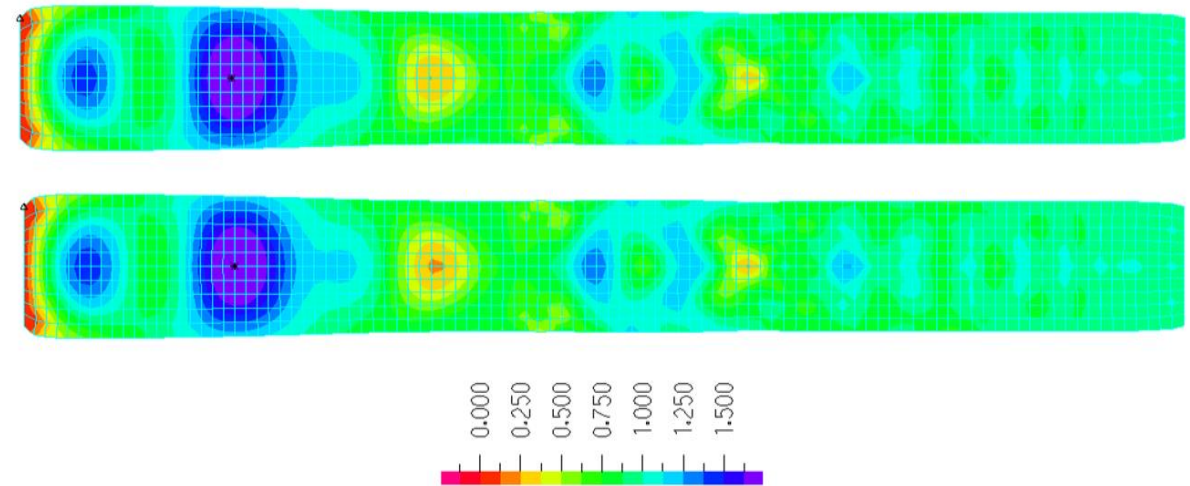

Fig. 1: Distributions of the velocity of wave propagation $[\mathrm{m} / \mathrm{s}]$ in Mooney - Rivlin material and Zahorski material for rubber "A" according to Table 1.

Step 90 - the top figure is Mooney - Rivlin material, the second is Zahorski material.
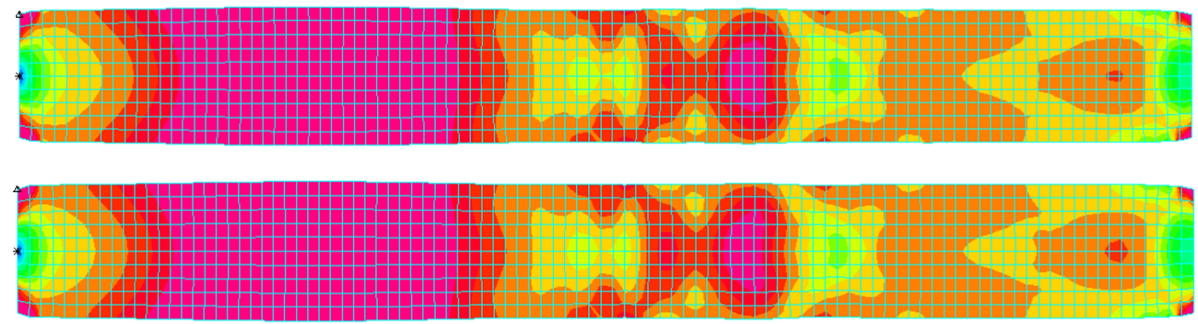

Step 100 - the top figure is Mooney - Rivlin material, the second is Zahorski material.
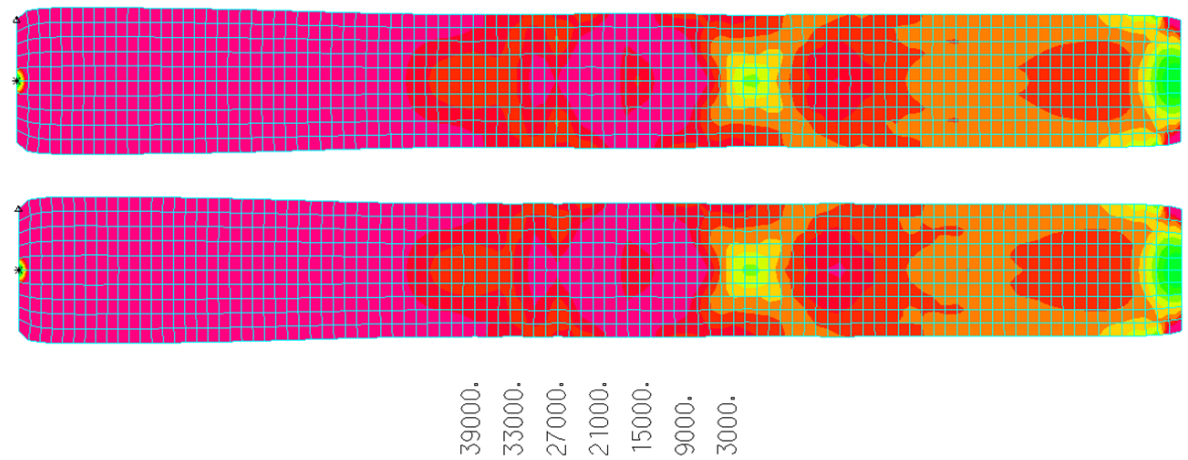

Fig. 2: Distributions of the effective stresses [Pa] in Mooney - Rivlin material and Zahorski material for rubber "A" according to Table 1. 


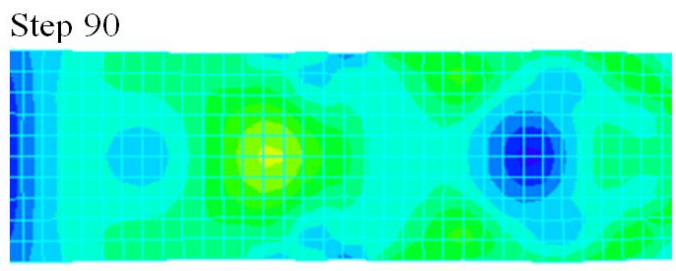

Step 100
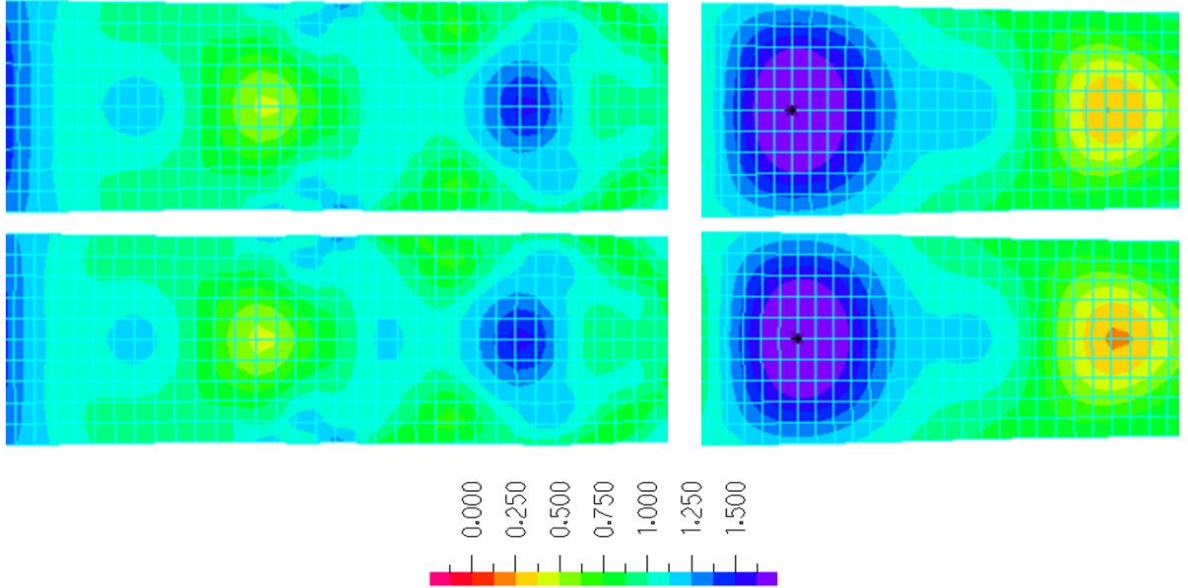

Fig. 3: Comparison of the distributions of propagation velocity $[\mathrm{m} / \mathrm{s}]$ for the rubber " $\mathrm{A}$ " according to Table 1. The uppermost material is Mooney - Rivlin material, the second is Zahorski material.

Step 90
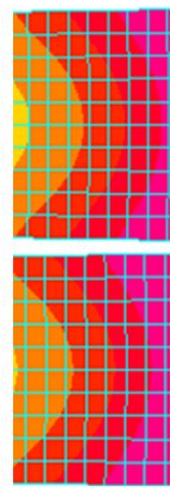

Step 100
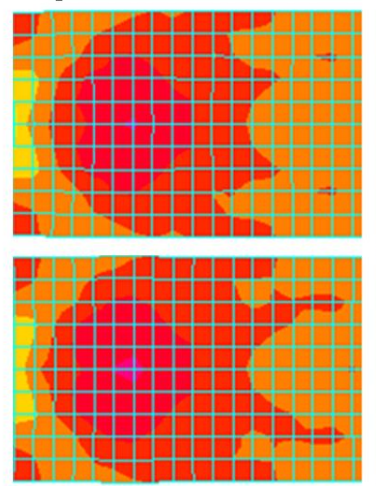
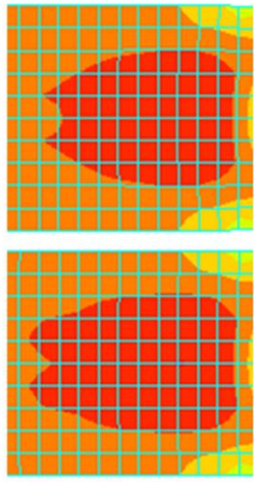

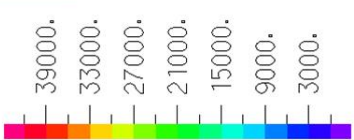

Fig. 4: Comparison of the states of effective stresses [Pa] for the rubber "A" according to Table 1. The uppermost material is Mooney - Rivlin material, the second is Zahorski material.

The comparative analysis of the velocity of wave (disturbance) propagation in Mooney - Rivlin and Zahorski material shows that the velocity of propagation of the wave in the rod ranges from $0 \mathrm{~m} / \mathrm{s}$ to $1.75 \mathrm{~m} / \mathrm{s}$. There are areas for the rod studied with noticeable differences in the values of velocity between the rod made of Mooney - Rivlin material and the rod made of Zahorski material. Analysis of wave propagation shows lower values of velocity of propagation in Zahorski material. Similarly, the differences in the distribution of the values of the effective stresses can be observed between the model of rod made of Mooney - Rivlin material and the model made of Zahorski material. The values of the effective stresses range from $0 \mathrm{~Pa}$ to $45.000 \mathrm{~Pa}$ and, for Zahorski material, the areas with lower values of stresses can be observed. The results obtained from model-based studies show insignificant qualitative and quantitative differences in distributions of stresses between Mooney-Rivlin material and Zahorski material since small differences occur between elastic potentials for both materials. Mooney-Rivlin material has two constants $C_{1}$ and $C_{2}$, linearly dependent on the invariants of the deformation tensor $I_{1}$ and $I_{2}$, whereas Zahorski material has three constants, with $\mathrm{C}_{1}$ and $\mathrm{C}_{2}$ being dependent linearly on the deformation tensor (similar to Mooney-Rivlin material), while the constant $C_{3}$ depends nonlinearly on the deformation tensor $I_{1}$. 


\section{References}

[1] MOONEY, M.: A theory of large deformations, J. Appl. Phys. 11, 1940, pp. 582-592.

[2] RIVLIN, R.S.: Large elastic deformations of isotropic materials, I Fundamental concepts., Phil. Trans. Roy. Soc. Lond. A 240, 1948, pp. 459-490.

[3] RIVLIN, R.S. - SAUNDERS, D.W.: Large elastic deformations of isotropic materials, VII Experiments of the deformation of rubber, Phil. Trans. Roy. Soc. Lond. 243, 1951, pp. 251-288.

[4] ZAHORSKI, S.: A form of elastic potential for rubber-like materials, Archives of Mechanics, 5, 1959, pp. 613-617.

[5] ZAHORSKI, S., Doświadczalne badania niektórych własności mechanicznych gumy, Rozprawy inżynierskie, tom 10 (1), 1962, pp. 193-207.

[6] KOSIŃSKI, S.: Fale sprężyste w gumopodobnych kompozytach warstwowych, Wydawnictwo Politechniki Łódzkiej, Łódź, 2007.

[7] KOSIŃSKI, S.: Odbicie i ewolucja fali uderzeniowej w wybranych materiałach hipersprężystych, Wydawnictwo IPPT PAN, Warszawa, 1995.

[8] MAJOR, M.: Velocity of Acceleration Wave Propagating in Hyperelastic Zahorski and MooneyRivlin Materials, J. Theoret. Appl. Mech.Vol. 43 nr 4, 2005, pp. 777-787, ISSN: 1429-2955.

[9] MAJOR, I. - MAJOR, M.: Traveling Waves in a Thin Layer Composed of Nonlinear Hyperelastic Zahorski's Material, J.Theoret.Appl.Mech. Vol. 47 nr 1, 2009, pp. 109-126, ISSN: 1429-2955.

[10]KLEPACZKO, J.: Doświadczalne badania sprężysto - plastycznych procesów falowych w metalach, IPPT PAN, Warszawa, 1970.

[11]MAJOR M.: Modelowanie zjawisk falowych w hipersprężystym materiale Zahorskiego, Wyd. P.Czest., Częstochowa, 2013, p.188, ISBN: 978-83-7193-600-5, ISSN: 0860-5017. 